\title{
CARACTERIZAÇÃO E PROPRIEDADES DO MATERIAL COLOIDAL NANOESTRUTURADO $\beta$-FeOOH/BENTONITA
}

\author{
Fauze Jacó Anaissi*, Juan Carlo Villalba e Sérgio Toshio Fujiwara \\ Departamento de Química, Setor de Ciências Exatas e de Tecnologia, Campus Cedeteg, Universidade Estadual do Centro-Oeste, \\ CP 3010, 85040-080 Guarapuava - PR, Brasil \\ Luiz Fernando Cótica \\ Departamento de Física, Setor de Ciências Exatas e de Tecnologia, Campus Cedeteg, Universidade Estadual do Centro-Oeste, \\ CP 3010, 85040-080 Guarapuava - PR, Brasil \\ Cláudio Roberto Lima de Souza e Patrício Zamora-Peralta \\ Departamento de Química, Centro Politécnico, Universidade Federal do Paraná, CP. 19081, 81531-990 Curitiba - PR, Brasil
}

Recebido em 15/6/08; aceito em 13/4/09; publicado na web em 17/9/09

\begin{abstract}
CHARACTERIZATION AND PROPERTIES OF COLLOIDAL $\beta$-FeOOH/BENTONITE NANOSTRUCTURED MATERIAL. A new mixed material was obtained through the combination of the suspensions of iron oxy-hydroxide and bentonite clay, denoted BFe. Analysis of its structure (XRD, Mossbauer and TGA) and composition (AAS) suggests the maintenance of the layer structure of the clay and an increase in the thermal stability of the BFe. Electrochemical studies performed in different electrolytes show that only in an alkaline medium it is possible to observe the redox peaks relative to the processes involving $\mathrm{Fe}^{+2} / \mathrm{Fe}^{+3}$ pair. Tests that evaluated the potential use of the photo-Fenton process showed an efficient degradation process of the dyes in significantly reduced reaction times.
\end{abstract}

Keywords: iron hydroxide; bentonite clay; sol-gel materials.

\section{INTRODUÇÃO}

Compostos à base de ferro têm sido amplamente utilizados desde os primórdios da nossa história, desempenhando um importante papel em inúmeras áreas de relevância. ${ }^{1}$ Embora muitas espécies sejam classificadas como óxidos de ferro, apenas a hematita $\left(\mathrm{Fe}_{2} \mathrm{O}_{3}\right)$, maghemita $\left(\gamma-\mathrm{Fe}_{2} \mathrm{O}_{3}\right)$ e magnetita são de fato óxidos, correspondendo às principais formas de óxido encontradas na natureza. Muitos hidróxidos, como hidróxido férrico $\left(\mathrm{Fe}(\mathrm{OH})_{3}\right)$, e oxi-hidróxidos, como a goetita $(\alpha-\mathrm{FeOOH})$ e a limonita $(\mathrm{FeOOH})$, também são de importância industrial, principalmente como precursores de óxidos puros ou complexos. ${ }^{1}$

De maneira geral, os óxidos e hidróxidos de ferro coloidal sempre foram objetos de estudo, o que proporcionou que algumas de suas fases fossem identificadas e caracterizadas. Essas fases compreendem um amplo grupo de compostos amorfos e cristalinos, que podem ser obtidos por vários métodos na forma de "óxidos férricos hidratados" ou "hidróxidos férricos". ${ }^{2}$ Assim, as estruturas e propriedades mais importantes de óxidos e hidróxidos de ferro têm sido discutidas e revisadas por vários grupos ao longo de décadas. ${ }^{2}$

Existem cerca de 17 óxidos, hidróxidos e oxi-hidróxidos de ferro, muitos dos quais são obtidos artificialmente. ${ }^{3}$ De maneira geral, estas espécies apresentam uma área de superfície muito grande e podem ser usados como agentes adsorventes e como catalisadores. Outros usos incluem a indústria de pigmentos, semicondutores, supercapacitores, dentre outros. ${ }^{1,3-4}$

Além de permitirem aplicações convencionais como as acima descritas, estes materiais podem ser utilizados na degradação oxidativa de substratos de relevância ambiental, por meio de processos Fenton (Equações 1-3). Em geral, admite-se que o sítio (三Fe(III)), representado na Equação 1, pode ser regenerado através da reação representada na Equação 3, fechando-se um ciclo catalítico que opera enquanto existir peróxido de hidrogênio. ${ }^{5}$ Em sistemas assistidos por

\footnotetext{
*e-mail: fauze.anaissi@pesquisador.cnpq.br
}

radiação (processos foto-Fenton), a forma $\equiv \mathrm{Fe}(\mathrm{III})$ é fotorreduzida a $\equiv \mathrm{Fe}$ (II) (Equação 4), o que favorece a decomposição do peróxido e a consequente formação de radical hidroxila. ${ }^{6}$

$$
\begin{aligned}
& \equiv \mathrm{Fe}(\mathrm{III})+\mathrm{H}_{2} \mathrm{O}_{2} \rightarrow \equiv \mathrm{Fe}(\mathrm{III}) \mathrm{H}_{2} \mathrm{O}_{2} \\
& \equiv \mathrm{Fe}(\mathrm{III}) \mathrm{H}_{2} \mathrm{O}_{2} \rightarrow \equiv \mathrm{Fe}(\mathrm{II})+\mathrm{HO}_{2} \cdot+^{+} \\
& \equiv \mathrm{Fe}(\mathrm{II})+\mathrm{H}_{2} \mathrm{O}_{2} \rightarrow \equiv \mathrm{Fe}(\mathrm{III})+{ }^{\circ} \mathrm{OH}+\mathrm{OH}^{-} \\
& \equiv \mathrm{Fe}(\mathrm{III})+\mathrm{H}_{2} \mathrm{O}+\mathrm{hv} \rightarrow \equiv \mathrm{Fe}(\mathrm{II})+\mathrm{H}^{+}+{ }^{\cdot} \mathrm{OH}
\end{aligned}
$$

Processos Fenton aplicados na presença de óxidos e oxihidróxidos de ferro têm sido abundantemente relatados na literatura recente, principalmente em estudos de degradação envolvendo espécies de relevância ambiental (ex. corantes têxteis). ${ }^{7-13}$ Em geral, os resultados atestam a potencialidade do processo, inclusive frente a espécies reconhecidamente resistentes em relação a outros tipos de tratamentos. Entretanto, a necessidade de se produzirem formas imobilizadas destes fotocatalisadores aparece como importante necessidade, principalmente para permitir o desenvolvimento de sistemas contínuos de tratamento.

Dente os inúmeros materiais propostos para a imobilização de catalisadores, é possível destacar as argilas lamelares, principalmente em função de características como elevada capacidade de troca iônica, alto grau de intumescimento, abundância e baixo custo. ${ }^{14-16}$ As argilas esmectitas, por exemplo, são aluminossilicatos lamelares, coloidais, aquosos e pertencem à classe dos filossilicatos. Essas argilas possuem uma combinação de propriedades de troca catiônica, de intercalação e intumescimento (ou expansão lamelar), que as tornam popular na química de intercalação hóspede-hospedeiro. ${ }^{17,18}$

Por sua vez, o processo sol-gel vem sendo utilizado cada vez mais na fabricação de novos materiais, especialmente, vidros, cerâmicas, filmes finos, materiais mistos e híbridos etc. Dentre as suas características intrínsecas, os géis inorgânicos apresentam propriedades 
de condução (iônica e eletrônica) e, consequentemente, atividade eletroquímica. ${ }^{19,20}$ Neste trabalho o ferro coloidal utilizado é produto derivado de uma propriedade de vários metais de transição em formar precipitados coloidais em solução alcalina. ${ }^{21,22}$

O presente trabalho teve por objetivo a preparação e caracterização das propriedades estruturais e eletroquímicas do gel de ferro, quando combinado com argilas do tipo montmorilonita. A caracterização estrutural foi baseada em difração de raios-X (DRX) e análise termogravimétrica (TGA), enquanto que a composição do material misto foi determinada por espectroscopia de absorção atômica (AAS). O comportamento eletroquímico foi verificado via voltametria cíclica e a sua potencialidade como fotocatalisador em processos foto-Fenton foi investigada frente a soluções aquosas de corantes têxteis.

\section{PARTE EXPERIMENTAL}

\section{Reagentes}

Os reagentes utilizados na produção do material em estudo foram: argila bentonita $\left(\mathrm{Al}_{2} \mathrm{O}_{3} \cdot \mathrm{Si}_{4} \mathrm{O}_{8} \cdot \mathrm{H}_{2} \mathrm{O}\right.$ - Aldrich); cloreto férrico hexa-aquo $\left(\mathrm{Fe}(\mathrm{OH})_{3} \cdot 6 \mathrm{H}_{2} \mathrm{O}\right.$ - Aldrich); hidróxido de amônio ( $\mathrm{NH}_{4} \mathrm{OH}$ - Biotec); ácido clorídrico ( $\mathrm{HCl}$ - Biotec); hidróxido de sódio ( $\mathrm{NaOH}$ - Reagen). Todos estes reagentes foram utilizados conforme recebidos, sem nenhum procedimento prévio de purificação. A atividade fotocatalítica dos materiais em estudo foi preliminarmente avaliada frente a soluções aquosas de corantes têxteis laranja reativo 16 (C.I. 17757, Aldrich) e preto reativo 5 (C.I. 20505, Aldrich), cuja estrutura é apresentada na Figura 1S (material suplementar). O peróxido de hidrogênio (Nuclear) foi utilizado em solução aquosa $(10 \% \mathrm{~m} / \mathrm{m})$, sendo que a sua concentração foi avaliada, quando necessária, por titulação permanganométrica.

Em todos os casos foi utilizada água deionizada de grau ultrapuro (Mili Q - $18 \Omega \mathrm{cm}^{-2}$ ).

\section{Preparação do hidróxido férrico coloidal - $\mathbf{F e}_{\mathrm{col}}$}

O gel de ferro foi preparado, via precipitação em meio básico, de forma similar ao método clássico de determinação de ferro via gravimétrica, com algumas adaptações para o nosso propósito. Para tanto, 6,0 g de cloreto férrico foram dissolvidos em $34 \mathrm{~mL}$ de ácido clorídrico (10,0 M), e o volume final, $200 \mathrm{~mL}$, completado com água. A solução foi aquecida até cerca de $80{ }^{\circ} \mathrm{C}$ por $30 \mathrm{~min}$ e, em seguida, adicionou-se lentamente hidróxido de amônio $(0,5 \mathrm{M})$ previamente aquecido $\left(80^{\circ} \mathrm{C}\right)$, até que não ocorresse mais precipitação de hidróxido férrico, $[\beta-\mathrm{FeOOH}], \mathrm{pH}$ 12,5. Após 48 h, quando todo o gel decantou, foi efetuada a lavagem do gel com água deionizada para remoção dos solúveis e diminuição do $\mathrm{pH}(8,0)$. Esse processo de lavagem por decantação e separação do sobrenadante demonstrou que o oxi-hidróxido férrico formado é bastante estável mesmo após a alteração do pH. Denominamos este oxi-hidróxido de $\mathrm{Fe}_{\mathrm{col}}$ (Oxi-hidróxido férrico coloidal).

\section{Preparação da bentonita}

A suspensão coloidal de argila bentonita (B - 2\%) foi preparada sob agitação por $24 \mathrm{~h}$ e mantida em repouso por uma semana para total maturação da suspensão. No momento da preparação do material misto, a suspensão foi agitada e mantida em repouso por $1 \mathrm{~h}$ para sedimentação das partículas maiores.

\section{Preparação do material misto coloidal - BFe}

Para a obtenção do material misto coloidal foram adicionadas, sob agitação constante, alíquotas do $\mathrm{Fe}_{\text {col. }}$ em uma suspensão de argila bentonita, na proporção de $5 \mathrm{~B} / 2 \mathrm{Fe}_{\mathrm{col}}(\mathrm{v} / \mathrm{v})$. Desta interação, formou-se um material gelatinoso de coloração amarelo-castanho que denominamos de BFe, óxido misto coloidal de argila Bentonita/ Ferro(III). A suspensão foi cuidadosamente decantada e lavada para remoção das partículas solúveis e do ferro coloidal em excesso. Repetiu-se esse processo várias vezes.

\section{Caracterização do BFe}

Para a caracterização estrutural e determinação da relação estequiométrica, o material misto $(\mathrm{BFe})$ e os dois materiais de partida (bentonita e $\mathrm{Fe}_{\text {col. }}$, estes com propósitos de comparação) foram secos à temperatura ambiente. Analise quantitativa do íon $\mathrm{Fe}^{3+}$ foi realizada em um espectrômetro de absorção atômica da Varian, modelo Spectra AA200, utilizando-se lâmpada de cátodo oco de ferro. Os espectros eletrônicos foram obtidos em um espectrofotômetro da Ocean Optics, modelo USB 2000, equipado com lâmpada de tungstênio/halogênio para comprimentos de onda entre 350-1050 nm e conjunto de fibra óptica (fonte-detector). Os difratogramas de raios- $\mathrm{X}$ foram obtidos em um difratômetro Rigaku, modelo Miniflex, equipado com lâmpada de cobre $(\lambda=1,54 \AA)$. As medidas de espectroscopia Mössbauer foram realizadas em temperatura ambiente, na geometria de transmissão, em um espectrômetro Mössbauer convencional no modo de aceleração constante e utilizando uma fonte de ${ }^{57} \mathrm{Co}(\mathrm{Rh})$ com uma atividade nominal de $10 \mathrm{mCi}$.Estudos eletroquímicos foram realizados em um potenciostato/galvanostato da BAS, modelo Epsilon, utilizando uma cela eletroquímica da BAS, modelo C3 Cell Stand, na configuração convencional com três eletrodos: eletrodo de trabalho um disco de platina, eletrodo de referência $(\mathrm{Ag} / \mathrm{AgCl})$ e fio de platina como eletrodo auxiliar. O eletrodo de trabalho foi utilizado de duas formas distintas, modificado e não modificado. A modificação do eletrodo de trabalho foi realizada pelo gotejamento da suspensão de BFe sobre a superfície da platina e deixado secar a temperatura ambiente por 5 min. Para propósitos de comparação foi realizada a voltametria cíclica para uma solução de cloreto férrico $\left(0,5 \mathrm{~mol} / \mathrm{dm}^{3}\right), \mathrm{pH} 7,5$. Em ambos os casos, o potencial aplicado nas varreduras foi entre os potenciais de -900 e $600 \mathrm{mV}$, nas velocidades de 50, 100, 150 e 200 $\mathrm{mV} \mathrm{s}^{-1}$. Como eletrólito suporte foi utilizada uma solução aquosa de $\mathrm{NaOH}\left(0,5 \mathrm{~mol} / \mathrm{dm}^{3}\right)$.

\section{Estudo de degradação de corantes por processos foto-Fenton}

A degradação da mistura de corantes têxteis (laranja reativo 16: $50 \mathrm{mg} \mathrm{L}^{-1}$ e preto reativo: $50 \mathrm{mg} \mathrm{L}^{-1}$ ) foi avaliada em um reator fotoquímico convencional de $300 \mathrm{~mL}$ de capacidade, equipado com agitação magnética e sistema de refrigeração por água. A radiação UV-A foi proporcionada por uma lâmpada a vapor de mercúrio de $125 \mathrm{~W}$ (sem o bulbo protetor), inserida na solução por meio de um bulbo de vidro Pyrex (radiação UV-A). Amostras de $250 \mathrm{~mL}$ tiveram o seu $\mathrm{pH}$ ajustado em valores previamente otimizados, sendo posteriormente submetidas a processo fotoquímico na presença de quantidades predeterminadas de peróxido de hidrogênio e catalisador suportado (BFe). Alíquotas foram coletadas em tempos convenientes e submetidas à análise espectrofotométrica, utilizando-se espectrofotômetro S-1150 (SCINCO) e cubetas de quartzo de 1,0 cm de caminho óptico.

\section{RESULTADOS E DISCUSSÃO}

\section{Análise composicional (AAS e TGA)}

Para realizar a quantificação estequiométrica, o material misto $\mathrm{BFe}$ foi seco a temperatura ambiente por $48 \mathrm{~h}$. Em seguida, foi digerido em $\mathrm{HNO}_{3}\left(0,1 \mathrm{~mol} \mathrm{dm}^{-3}\right)$ a $80{ }^{\circ} \mathrm{C}$ por $1 \mathrm{~h}$ em recipiente fechado; as soluções obtidas foram transferidas para balões volumétricos e 
seu volume completado com água. Para definir a composição dos materiais, por conveniência, expressamos a mesma em termos de argila bentonita e oxi-hidróxido de Ferro(III), assumindo as fórmulas estequiométricas aproximadas $\mathrm{Al}_{2} \mathrm{O}_{3} \cdot \mathrm{Si}_{4} \mathrm{O}_{8} \cdot \mathrm{H}_{2} \mathrm{O}(\mathrm{MM}=360,31 \mathrm{~g} / \mathrm{mol})$ e $\beta$-FeOOH $(\mathrm{MM}=106,86 \mathrm{~g} / \mathrm{mol})$, respectivamente. As quantidades relativas foram obtidas a partir de análise por espectroscopia de absorção atômica (AAS) da quantidade de $\mathrm{Fe}^{3+}$ presente na amostra. Assim, de acordo com os dados de AAS, o BFe pode ser formulado como $\mathrm{Al}_{2} \mathrm{O}_{3} \cdot \mathrm{Si}_{4} \mathrm{O}_{8} \cdot[\mathrm{\beta}-\mathrm{FeOOH}]_{\mathrm{r}} \cdot \mathrm{yH} \mathrm{H}_{2} \mathrm{O}$, onde $\mathrm{x}=0,48$ (determinado por AAS) e y =2,1 - 2,5 (determinado por TGA). Ou seja, a composição estimada do material misto $\mathrm{BFe}$ pode ser expressa como $\mathrm{Al}_{2} \mathrm{O}_{3} \cdot \mathrm{Si}_{4} \mathrm{O}_{8}$. $[\beta-\mathrm{FeOOH}]_{0,48} \cdot 2,3 \mathrm{H}_{2} \mathrm{O}$. Essa composição poderá facilitar eventuais cálculos quantitativos de rendimento quando o $\mathrm{BFe}$ for utilizado como catalisador ou agente promotor de degradação.

\section{Difração de raios-X (DRX)}

$\mathrm{O}$ padrão de difração de raios-X para o material $\mathrm{BFe}$ apresenta uma estrutura lamelar típica de um sólido bidimensional, e é comparável com o observado para a argila bentonita (Figura 1). A distância interlamelar de $15,53 \AA$ foi calculada pelo pico de difração 001 , distância essa maior que o $15,05 \AA$ da bentonita, sugerindo um processo de expansão lamelar devido à incorporação do $\mathrm{Fe}_{\text {col }}$ substituindo moléculas de água. Destacam-se, ainda, os picos 003 com 14,41 e 15,27 $\AA$ e 005 com 15,68 e 15,10 ̊, ambos, respectivamente, para a argila bentonita e para o $\mathrm{BFe}$. Outros picos são destacados por apontarem a presença de pequenos cristalitos da argila em $21,9^{\circ}(4,03 \AA)$, assim como os picos em $26,66^{\circ}(3,31 \AA), 33,16^{\circ}$ (2,66 ̊) e $35,60^{\circ}(2,48 \AA)$ para o $\mathrm{BFe}$, atribuídos ao processo de intercalação ocorrido na argila bentonita. Esses picos são típicos da fase Akaganeíta $(\beta-\mathrm{FeOOH}){ }^{3,4}$ Destacamos que o material deve ser bastante amorfo, devido ao método de síntese e ao tamanho das partículas geradas.

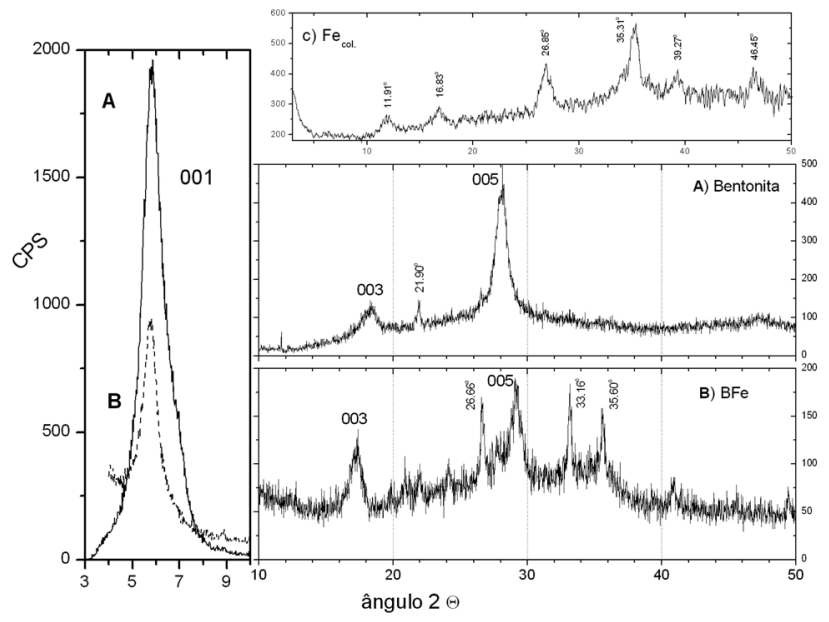

Figura 1. Difratograma de raios-X para: a) argila bentonita; b) material misto BFe e c) ferro coloidal

\section{Espectroscopia eletrônica (UV-Vis)}

Na Figura 2, são mostrados os espectros eletrônicos para o ferro coloidal e para o material misto BFe. Observa-se que o espectro do $\mathrm{Fe}_{\text {col }}$ apresenta duas bandas características em 500 e $900 \mathrm{~nm}$; a primeira é devida à transferência de carga metal-ligante e a segunda é devida possivelmente ao ligante aquo ou grupamento hidroxila $\left(\mathrm{OH}^{-}\right)$; ambas são típicas de complexos octaédricos do tipo $\left[\mathrm{Fe}\left(\mathrm{H}_{2} \mathrm{O}\right)_{6}\right]^{2+} \cdot{ }^{4} \mathrm{No}$ espectro do $\mathrm{BFe}$ além dessas duas bandas ocorre o aparecimento de uma terceira banda em $700 \mathrm{~nm}$, que pode ser atribuída a transições de campo ligante (d-d) do metal. Essa banda indica que o processo de interação entre argila bentonita e oxi-hidróxido férrico é mais que uma mistura física, conduz a interações mais especificas com mudança de simetria da esfera de coordenação do íon férrico. ${ }^{4}$

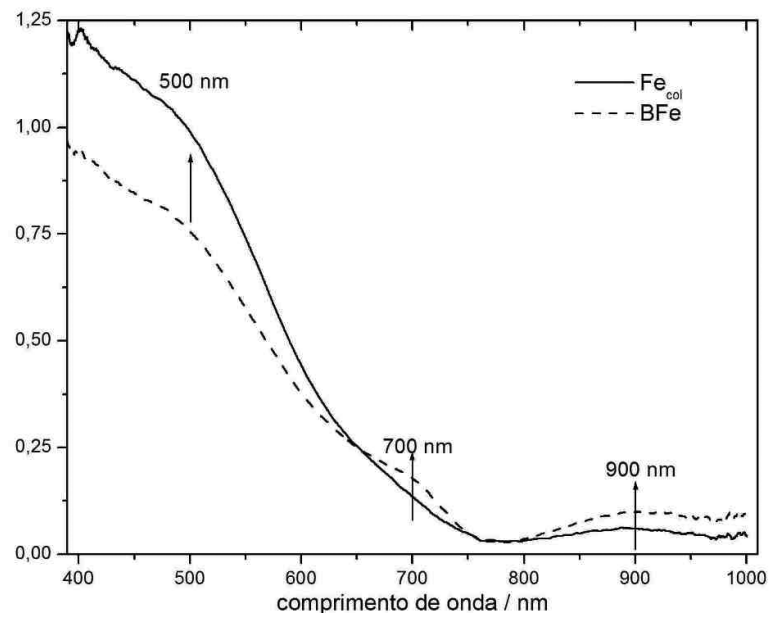

Figura 2. Espectros eletrônicos por reflectância difusa para ferro coloidal e para material misto $\mathrm{BFe}$

\section{Espectroscopia Mössbauer}

Na Figura 2S (material suplementar) são mostrados os espectros Mössbauer obtidos para a argila pura, para o $\mathrm{Fe}_{\text {col }}$ e para o material misto (BFe), todos na forma de pó obtidos por secagem a temperatura ambiente. Os espectros Mössbauer foram analisados através de uma rotina que utilizou linhas espectrais no formato de linhas Lorentzianas. Os valores obtidos para o deslocamento isomérico $(\delta)$ são relativos ao $\beta-\mathrm{FeOOH}$.

Os resultados (parâmetros hiperfinos) obtidos através do ajuste dos espectros Mössbauer são apresentados na Tabela 1. O espectro Mössbauer da argila pura mostra a presença residual de ferro em dois estados de oxidação diferentes, ou seja, íons $\mathrm{Fe}^{2+} \mathrm{e} \mathrm{Fe}^{3+}$. $\mathrm{O}$ espectro obtido no pó de $\mathrm{Fe}_{\text {col }}$ apresentou um único componente relativo ao íon $\mathrm{Fe}^{3+}$. O espectro do material misto apresenta dois componentes distintos relativos ao íon $\mathrm{Fe}^{3+}$. O componente majoritário (88\%, sítio 1) pode ser associado ao $\beta-\mathrm{FeOOH}$ que não reagiu com a bentonita, enquanto que o componente minoritário (12\%, sítio 2) pode ser associado à formação do material misto e estar relacionado com os picos dos cristalitos apontados no DRX, ou seja, à formação da fase akaganeíta.

\section{Comportamento eletroquímico (VC)}

Na Figura 3 e 3S (material suplementar), são apresentados os voltamogramas cíclicos para o eletrodo de platina modificado com BFe e para uma solução aquosa de $\mathrm{FeCl}_{3}$ em pH 7,5. Na Figura 3S está mostrado o comportamento eletroquímico para a solução aquosa de $\mathrm{FeCl}_{3}$; os VC apresentam um pico de redução em $320 \mathrm{mV}$ associado com o par redox $\mathrm{Fe}^{\mathrm{II}} / \mathrm{Fe}^{\mathrm{III}}$. Neste meio $(\mathrm{pH} 7,5)$ ocorre a formação de uma pequena quantidade de hidróxido de ferro(III) e a sua presença reflete no aparecimento do par redox na região entre -600 e $-300 \mathrm{mV}$ associada aos processos de redução envolvendo o grupamento $\mathrm{OH}^{-}$(hidroxila), ou seja, uma fração do aquocomplexo $\left.\left[\mathrm{Fe}\left(\mathrm{H}_{2} \mathrm{O}\right)_{6}\right)\right]^{3+}$ é convertido em $\left.\left[\mathrm{Fe}-\mathrm{OH}_{\mathrm{n}}\left(\mathrm{H}_{2} \mathrm{O}\right)_{6-\mathrm{n}}\right)\right]^{3+}$ envolvendo assim a reação abaixo [3]:

$$
1 / 2 \mathrm{Fe}_{2} \mathrm{O}_{3} \cdot 3 \mathrm{H}_{2} \mathrm{O}+\mathrm{e} \leftrightarrow \mathrm{Fe}(\mathrm{OH})_{2(\mathrm{~s})}+\mathrm{OH}^{-} \quad \mathrm{E}^{0}=-560 \mathrm{mV}
$$

Por outro lado, para o eletrodo modificado com BFe (Figura 3) esse efeito é intensificado e o potencial redox é deslocado para regiões 
mais catódicas, ou seja, o par devido à redução dos ligantes hidroxilas $\left(\mathrm{OH}^{-}\right)$concentra-se na região entre -900 e $-800 \mathrm{mV}$, efeito esse refletido também no pico redox do par $\mathrm{Fe}^{\mathrm{II}} / \mathrm{Fe}^{\mathrm{III}}$. Esse deslocamento de potencial implica numa diminuição da estabilidade termodinâmica, tornando o sistema $\mathrm{BFe}$ mais oxidante que o $\mathrm{Fe}(\mathrm{OH})_{3}$. Essa atribuição, considerada inicial, será melhor interpretada em termos de comportamento eletroquímico considerando soluções eletrolíticas em função do cátion $\left(\mathrm{Li}^{+}, \mathrm{Na}^{+}\right.$e $\left.\mathrm{K}^{+}\right)$e do ânion $\left(\mathrm{OH}^{-}\right.$e outros $)$, bem como da sua concentração.

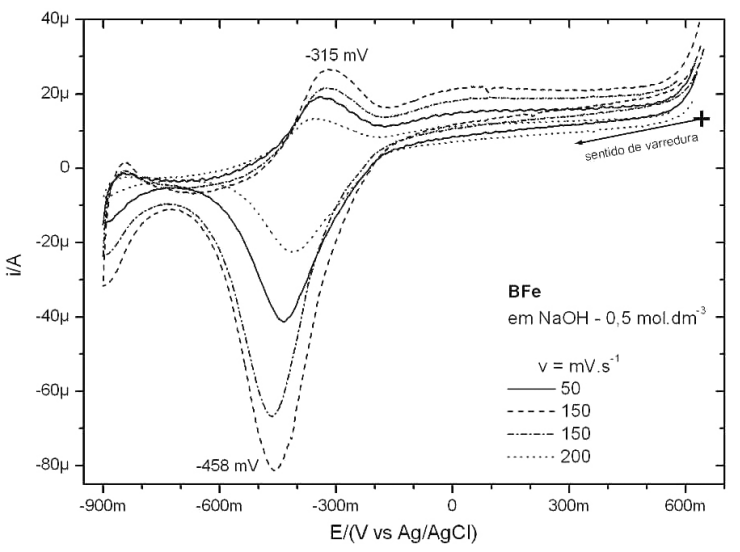

Figura 3. Voltametria cíclica para o eletrodo modificado com BFe em eletrólito suporte alcalino $\left(\mathrm{NaOH} 0,5 \mathrm{~mol} \mathrm{dm}^{-3}\right)$. Soluções eletrolíticas deaeradas com $\mathrm{N}_{2}$ por 15 min para remoção de $\mathrm{O}_{2}$ dissolvido

\section{Degradação de corantes pelo processo foto-Fenton}

Estudos preliminares (não publicados) têm permitido constatar uma elevada potencialidade de formas férricas imobilizadas em resinas de troca iônica e zeólitas, para aplicação em processos de degradação do tipo like-Fenton. Infelizmente, a elevada reatividade destes suportes faz com que, em muitos casos, a sua elevada capacidade de adsorção mascare os processos de degradação estudados. Para contornar este inconveniente, propôs-se a utilização de um suporte menos reativo (argila) e a sua modificação com Ferro (III) na forma de $\mathrm{BFe}$.

Trabalhando-se nas condições previamente otimizadas (resultados não apresentados), estudos de degradação da mistura de corantes foram realizados obtendo-se os resultados apresentados na Figura 4. Nesta sequência de espectros é possível observar uma rápida degradação dos cromóforos, o que permite uma completa remoção da cor nos primeiros 10 min de reação. Adicionalmente, observa-se uma eficiente degradação da fração aromática da molécula, que absorve fortemente entre 250 e $300 \mathrm{~nm}$, após $30 \mathrm{~min}$. De maneira geral, admite-se que a degradação de espécies de caráter aromático se inicia com a adição de radical hidroxila, o que leva à geração de intermediários fenóli- cos. Posteriormente o anel benzênico hidroxilado é clivado, com a formação de ácidos carboxílicos de cadeia curta. ${ }^{23}$ Desta forma, o sinal residual observado em $200 \mathrm{~nm}$ deve corresponder aos ácidos mucônico e oxálico, que tendem a acumular no final do processo. ${ }^{24}$

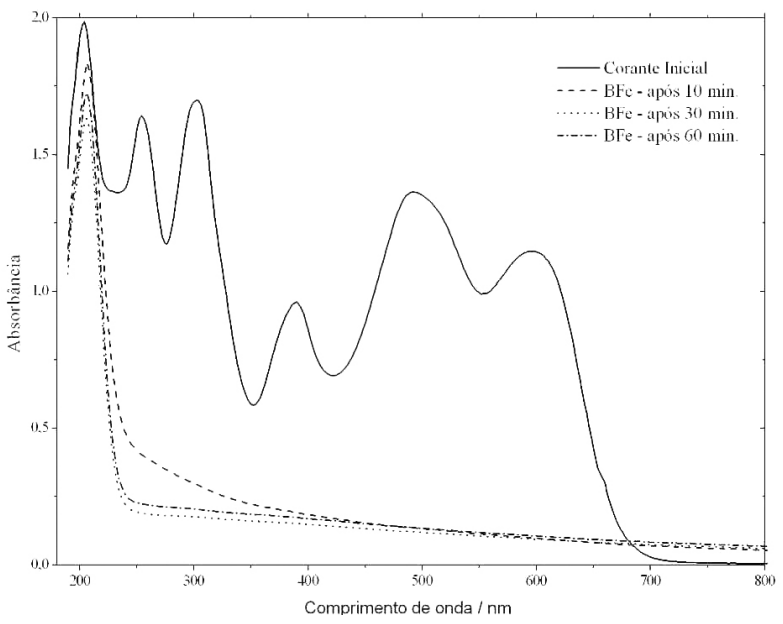

Figura 4. Monitoramento espectroscópico da solução de corantes: laranja reativo 16 e preto reativo 5 (100 $\left.\mathrm{mg} \mathrm{L}^{-1} \mathrm{c} / \mathrm{u}\right)$; $\mathrm{pH}: 3,0$, suporte: $33 \mathrm{mg}$. BFe degradação da mistura via processo Fenton

Em função da natureza do sistema fotoquímico empregado, a diminuição dos parâmetros monitorados pode ser o resultado de três processos concomitantes: adsorção do substrato na superfície da argila; decomposição por fotólise e decomposição pelo sistema $\mathrm{H}_{2} \mathrm{O}_{2} / \mathrm{UV}-\mathrm{A}$. O processo de fotólise provoca certas modificações estruturais no corante, o que se manifesta como aumento no valor de absorbância. Entretanto, a experiência sugere que tais modificações sejam bastante leves, envolvendo principalmente a quebra de ligações mais lábeis. Estudos de degradação de corantes por fotólise (ação isolada da luz) têm demonstrado a formação de uma complexa mistura de fotoprodutos, com destaque para formas desmetiladas e hidroxiladas. ${ }^{25}$

Embora a decomposição fotoquímica do peróxido de hidrogênio, e a consequente geração de radical hidroxila, se processe com radiações da ordem de $254 \mathrm{~nm}$, descolorações de até $20 \%$ podem ser induzidas pelo sistema assistido por radiação UV-A.

Finalmente, o processo de adsorção foi completamente inexpressivo em relação à remoção do corante, quando se utiliza apenas a argila bentonita, Figura 4S (material suplementar). Trata-se de um antecedente de extrema relevância, uma vez que grande parte dos materiais propostos como suporte de catalisadores (ex., carvão ativado, resinas de troca iônica etc.) apresenta uma elevada capacidade de adsorção, o que faz com que grande parte da remoção observada seja devida, apenas, a processos de separação física (adsorção).

Tabela 1. Parâmetros hiperfinos obtidos através do ajuste dos espectros Mössbauer

\begin{tabular}{|c|c|c|c|c|c|}
\hline Amostra & Sub-espectro & $\begin{array}{c}\delta^{*}(\mathrm{~mm} / \mathrm{s}) \\
( \pm 0,02)\end{array}$ & $\begin{array}{c}\text { QS }(\mathrm{mm} / \mathrm{s}) \\
( \pm 0,02)\end{array}$ & $\begin{array}{l}\text { Área }(\%) \\
\quad( \pm 1)\end{array}$ & $\begin{array}{c}\text { FWHM } \\
(\mathrm{mm} / \mathrm{s})\end{array}$ \\
\hline \multirow{2}{*}{ Bentonita } & $\mathrm{Fe}^{3+}$ & 0,29 & 0,68 & 68 & 0,60 \\
\hline & $\mathrm{Fe}^{2+}$ & 1,51 & 2,77 & 32 & 0,80 \\
\hline$\beta-\mathrm{FeOOH}$ & $\mathrm{Fe}^{3+}$ & 0,35 & 0,67 & 100 & 0,44 \\
\hline \multirow{2}{*}{$\mathrm{BFe}$} & $\mathrm{Fe}^{3+}$ sítio 1 & 0,33 & 0,70 & 88 & 0,40 \\
\hline & $\mathrm{Fe}^{3+}$ sítio 2 & 0,40 & 0,40 & 12 & 0,40 \\
\hline
\end{tabular}

${ }^{*}$ Relativo ao $\alpha$-Fe em temperatura ambiente. 


\section{CONCLUSÃO}

A interação entre as suspensões aquosas de hidróxido de ferro(III) e argila bentonita gera um material misto com comportamento eletroquímico muito atrativo, pois combina a capacidade de troca iônica da argila com a rica atividade eletroativa do ferro. Os resultados preliminares mostram que a interação envolve mais que uma simples mistura física. A espectroscopia eletrônica destaca o aparecimento de uma banda de transições de campo ligante (d-d), não conferida ao gel de ferro(III). Por outro lado, o comportamento eletroquímico comparativo para íons férrico em solução e do eletrodo modificado com o BFe mostra que o pico catódico devido ao processo $\mathrm{Fe}$ (II)/Fe(III) deslocou do potencial inicial em 320 para $-458 \mathrm{mV}$, enquanto que o processo relativo à redução envolvendo o grupamento $\mathrm{OH}^{-}$(hidroxila) deslocou de -585 para -900 $\mathrm{mV}$, o que atribuímos como sendo uma diminuição da estabilidade termodinâmica, tornando o sistema $\mathrm{BFe}$ mais oxidante que o $\beta-\mathrm{FeOOH}$.

Avaliou-se ainda a potencialidade do processo foto-Fenton frente à degradação de corantes reativos, utilizando-se o BFe. A argila como suporte apresentou ótimas condições para imobilização de óxido férrico, assim como para servir de fonte de Ferro(III) para processos do tipo Fenton. O sistema permitiu uma eficiente degradação de corantes reativos em tempos de reação bastante reduzidos, o que sugere uma boa potencialidade para a eliminação de cor em resíduos têxteis.

\section{MATERIAL SUPLEMENTAR}

No material suplementar, disponível em http://quimicanova.sbq. org.br na forma de arquivo PDF, com acesso gratuito, estão apresentadas as Figuras $1 \mathrm{~S}$ a 4S. Na Figura $1 \mathrm{~S}$ estão representadas as fórmulas estruturais dos corantes laranja reativo 16 (Aldrich) e preto reativo 5 (Aldrich). Na Figura 2S tem-se os espectros Mössbauer para os materiais de partida bentonita e $\mathrm{Fe}_{\mathrm{col}}$ e para o material coloidal misto BFe. De maneira completar e com propósitos de comparação, a Figura $3 \mathrm{~S}$ apresenta a voltametria cíclica para uma solução aquosa de $\mathrm{FeCl}_{3}$ $\left(0,5 \mathrm{~mol} \mathrm{dm}^{-3}\right)$ em meio alcalino $(\mathrm{pH} 7,5)$. A Figura $4 \mathrm{~S}$ apresenta o monitoramento espectroscópico da solução de corantes (laranja reativo 16 e preto reativo 5) realizada apenas para a argila bentonita.

\section{AGRADECIMENTOS}

Ao apoio financeiro do CNPq (Edital Universal, processo: 473446/2003-0), da Fundação Araucária (Edital Recém-Doutor, processo: 4642) e da Renami-CNPq, e ás medidas de espectroscopia Mössbauer fornecidas pelo Prof. A. Paesano Jr. (UEM). J. C. Villalba agradece a bolsa de Iniciação Científica, vinculada ao programa PBPI/ UNICENTRO e a bolsa de Mestrado DS-Capes.

\section{REFERÊNCIAS}

1. Senna, M.; Dekker Encyplopedia of Nanoscience and Nanotechnology 2004, p. 1525.

2. Towe, K. M.; Bradley, W. F.; J. Colloid Interface Sci. 1967, 24, 384

3. Cai, J.; Liu, G.; Gao, Z.; Navrotsky, A.; Suib, S. L.; Chem. Mater. 2001, 13,4595 .

4. Schwertmann, U.; Cornell, R. M.; The Iron Oxides, $2^{\text {nd }}$ ed., John Wiley: New York, 2003, cap. 1, 7 e 20.

5. Dantas, T. L. P.; Mendonça,V. P.; José, H. J.; Rodrigues, A. E.; Moreira, R. F. P. M.; Chem. Eng. J. 2006, 118, 77.

6. Hsueh, C. L.; Huang, Y. H.; Wang, C. C.; Chen, C. Y., J. Mol. Catal. A: Chem. 2006, 245, 78 .

7. Gumy, D.; Fernández-Ibánez, P.; Malato, S.; Pulgarin, C.; Enea, O.; Kiwi, J.; Catal. Today 2005, 101, 375.

8. Hsueh, C. L.; Lu, Y. W.; Hung, C. C.; Huang, Y. H.; Chen, C. Y.; Dyes Pigm. 2007, 75, 130.

9. Hsueh, C. L.; Huang, Y. H.; Wang, C. C.; Chen, C. Y.; J. Mol. Catal. A: Chem. 2006, 245, 78 .

10. Laine, D. F.; Cheng, I. F.; Microchem. J. 2007, 85, 183.

11. Martinez, F.; Calleja, G.; Melero, J. A.; Molina, R.; Appl. Catal. B 2007, $70,452$.

12. Hsueh, C. L.; Huang, Y. H.; Chen, C. Y.; J. Hazard. Mater. 2006, 129, 228.

13. Lv, X.; Xu, Y.; Lv, K.; Zhang, G.; J. Photochem. Photobiol. A 2005, 173, 121.

14. Doff, D. H.; Gangas, N. H. J.; Allan, J. E. M.; Coey, J. M. D.; Clay Miner. 1988, 23, 367 .

15. Choudary, B. M.; Sarma, M. R.; Kumar, K. V.; J. Mol. Catal. 1994, 98 , 101.

16. Wang, S.; Zhu, H. Y.; Lu, G. Q.; J. Colloid Interface Sci. 1998, 204, 128.

17. Pergher, S. B. C.; Corma, A.; Forners, V.; Quim. Nova 1999, $22,693$.

18. Coelho, A. C. V.; Santos, P. S.; Santos, H. S.; Quim. Nova 2007, 30, 146; Coelho, A. C. V.; Santos, P. S.; Santos, H. S.; Quim. Nova 2007, 30,1282 .

19. Livage, J.; Henry, M.; Sanchez, C.; Prog. Solid. State Chem. 1988, 18, 259.

20. Bard, A. J.; J. Chem. Educ. 1983, 60, 302.

21. Lee, J. D.; Química Inorgânica Não Tão Concisa, $3^{\mathrm{a}}$ ed., Ed. Edgard Blücher Ltda: São Paulo, 1980.

22. Cotton, F. A.; Wilkinson, G.; Advanced Inorganic Chemistry, Jonh Wiley: New York, 1980.

23. Du, Y.; Zhou, M.; Lei, L.; J. Hazard. Mater. 2007, 139, 108.

24. Du, Y.; Zhou, M.; Lei, L.; Water Res. 2007, 41, 1121.

25. Pérez-Estrada, L. A.; Agüera, A.; Hernando, M. D.; Malato, S.; Fernández-Alba, A. R.; Chemosphere 2008, 70, 2068. 


\section{CARACTERIZAÇÃO E PROPRIEDADES DO MATERIAL COLOIDAL NANOESTRUTURADO $\beta$-FeOOH/}

\section{BENTONITA}

Fauze Jacó Anaissi*, Juan Carlo Villalba e Sérgio Toshio Fujiwara

Departamento de Química, Setor de Ciências Exatas e de Tecnologia, Campus Cedeteg, Universidade Estadual do Centro-Oeste, CP 3010, 85040-080 Guarapuava - PR, Brasil

\section{Luiz Fernando Cótica}

Departamento de Física, Setor de Ciências Exatas e de Tecnologia, Campus Cedeteg, Universidade Estadual do Centro-Oeste, CP 3010, 85040-080 Guarapuava - PR, Brasil

Cláudio Roberto Lima de Souza e Patrício Zamora-Peralta

Departamento de Química, Centro Politécnico, Universidade Federal do Paraná, CP. 19081, 81531-990 Curitiba - PR, Brasil

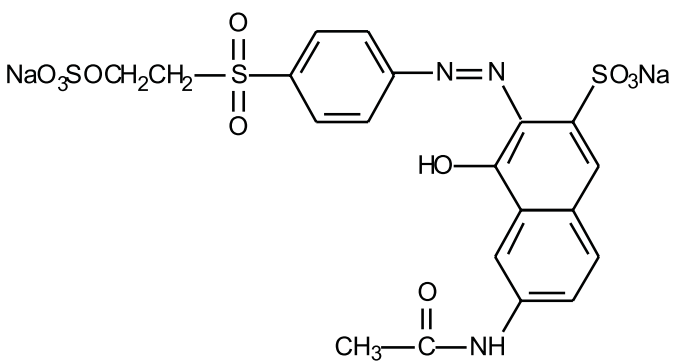

Reactive Orange 16 (C.I.Nㅜ: 17757)

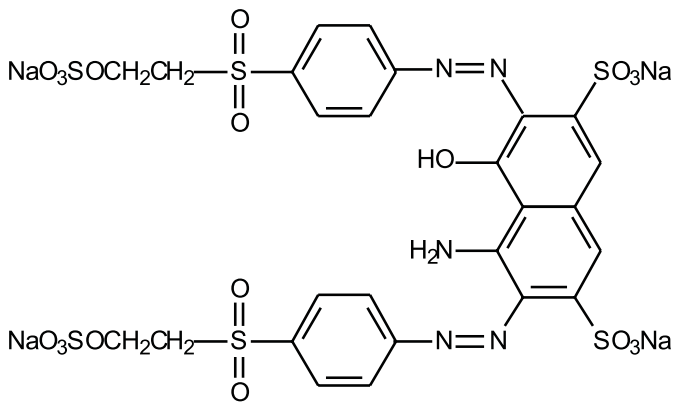

Reactive Black 5 (C.I. №: 20505)

Figura 1S. Fórmula estrutural dos corantes laranja reativo 16 (Aldrich) e preto reativo 5 (Aldrich)

*e-mail: fauze.anaissi@pesquisador.cnpq.br 


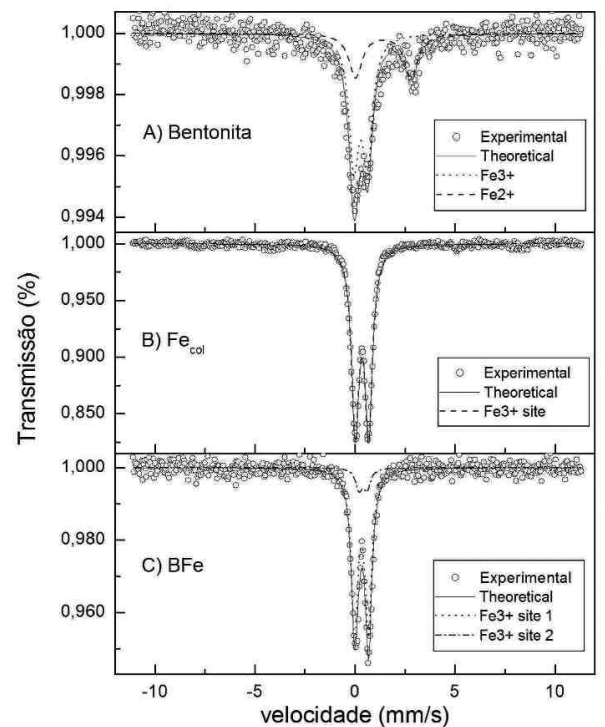

Figura 2S. Espectros Mössbauer para os materiais de partida bentonita e $\mathrm{Fe}_{\text {col }}$ e para o material coloidal misto BFe

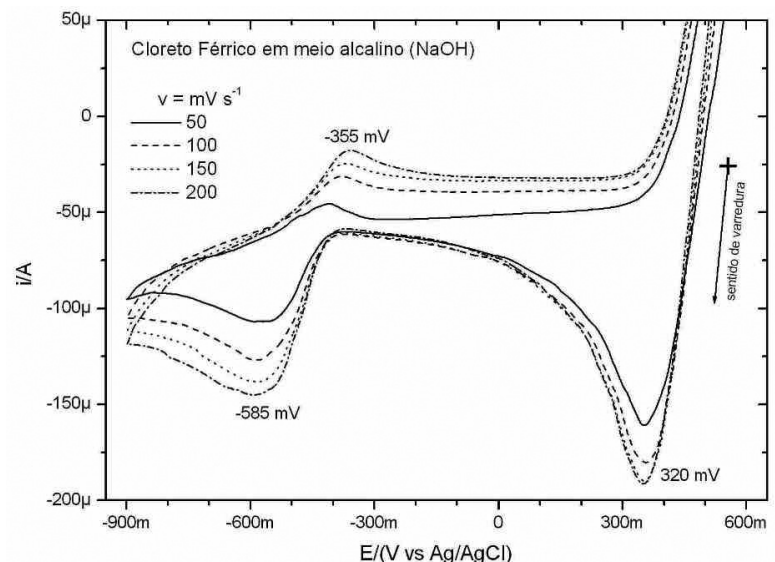

Figura 3S. Voltametria cíclica para uma solução aquosa de $\mathrm{FeCl}_{3}\left(0,5 \mathrm{~mol} \mathrm{dm}^{-3}\right)$ em meio alcalino $(p H \mathrm{H}, 5)$. Soluções eletrolíticas deaeradas com $\mathrm{N}_{2}$ por 15 min para remoção de $\mathrm{O}_{2}$ dissolvido

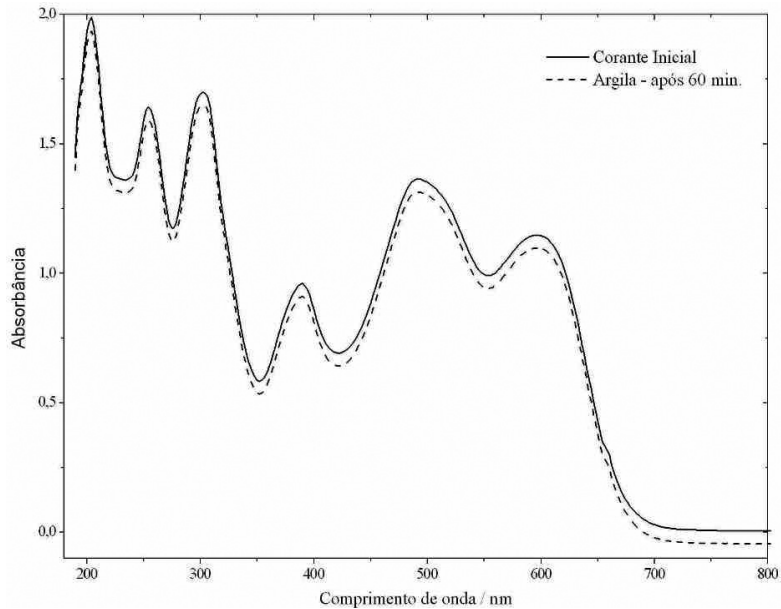

Figura 4S. Monitoramento espectroscópico da solução de corantes laranja reativo 16 e preto reativo 5 (100 mg $\mathrm{L}^{-1}$ c/u); pH: 3,0, suporte: $33 \mathrm{mg}$. Argila bentonita - adsorção dos corantes 